University of Nebraska - Lincoln

DigitalCommons@University of Nebraska - Lincoln

\title{
Basic materials research programs at the U.S. Air Force Office of Scientific Research
}

Herbert C. Carlson

Air Force Office of Scientific Research

K. C. Goretta

Air Force Office of Scientific Research

Follow this and additional works at: https://digitalcommons.unl.edu/usafresearch

Part of the Aerospace Engineering Commons

Carlson, Herbert C. and Goretta, K. C., "Basic materials research programs at the U.S. Air Force Office of Scientific Research" (2006). U.S. Air Force Research. 18.

https://digitalcommons.unl.edu/usafresearch/18

This Article is brought to you for free and open access by the U.S. Department of Defense at DigitalCommons@University of Nebraska - Lincoln. It has been accepted for inclusion in U.S. Air Force Research by an authorized administrator of DigitalCommons@University of Nebraska - Lincoln. 


\title{
Basic materials research programs at the U.S. Air Force Office of Scientific Research
}

\author{
Herbert C. Carlson*, K.C. Goretta \\ Air Force Office of Scientific Research, Arlington, VA 22203-1768, USA
}

\begin{abstract}
The Air Force Office of Scientific Research (AFOSR) annually sponsors approximately 5000 research scientists at 1000 universities and laboratories, generating about 10,000 Ph.D. graduates per decade, all expected to publish their basic research findings in peer-reviewed journals. After a brief introduction of the nature of AFOSR's support to basic research in the U.S. and international scientific communities, work it supports at the frontiers of materials science is highlighted. One focused research theme that drives our investment is the MEANS program. It begins with the end in mind; materials are designed with practicable manufacture as an explicit initial goal. AFOSR's broad research portfolio comprises many materials. Nanotechnology efforts include optical materials that reduce distortion to the scale of the nanoparticles themselves. Advances in semiconductors include breakthroughs in Group III nitrides, some of which emanated from Asia under sponsorship from AFOSR's Asian office. Advances in structural materials include those for use at ultra-high temperatures and self-healing composites. The growing role of high-performance computing in design and study of functional, biological, and structural materials is also discussed.
\end{abstract}

Published by Elsevier B.V.

Keywords: Ceramics; Laser processing; Metals; Optical properties

\section{Introduction}

The Air Force Office of Scientific Research (AFOSR) is the sole manager of basic research for the U.S. Air Force (USAF). It is part of the Air Force Research Laboratory (AFRL) [1]. With its approximately US\$ $210 \mathrm{M}$ in core funding, plus other funds from programs such as the multi-university research initiative (MURI) and small business technology transfer (STTR) program [2], AFOSR supports about 5000 researchers in universities, industry, and AF laboratories, generating about 10,000 $\mathrm{Ph} . \mathrm{D}$. graduates per decade in science and technology areas within its broad technical scope. Its research is managed by a small core of experienced, technically strong, scientifically accomplished researchers with in-depth knowledge of their respective fields of science. In addition to extensive support to the national research community, it also does, as it must, seek the best researchers internationally to support the best research it finds in areas of mutual interest. As for all basic research it supports, AFOSR has the expectation that any work that it funds will stand up to peer review and be published in the open literature.

\footnotetext{
* Corresponding author. Tel.: +1 703696 7556; fax: +1 7036969556.

E-mail address: herb.carlson@ afosr.af.mil (H.C. Carlson).
}

Because of the very nature of the broad span of research we support, here we can only highlight representative elements of our research programs, restricting considerations to materials-related work. The sections below seek to accomplish this overview through discussions of key programs and projects in functional and structural materials and materials modeling.

\section{Materials engineering for affordable new systems: MEANS}

Many potentially highly valuable materials have been discovered and developed, only to go unused for want of building in manufacturability from the start. Others have taken longer to implement, and been more costly than necessary, for want of planning in manufacturability from the start. The MEANS program (from materials engineering for affordable new systems) is an initiative by AFOSR, designed to confront this issue and to take advantage of the capabilities of simulation and modeling to guide and focus research and development efforts [3]. The objective of the MEANS program is to develop a global design methodology to integrate materials modeling and strategic experimentation (Fig. 1). Its main features are: it spans length and time scales; it emphasizes compatibility as adjacent scales; it functions effectively with existing design software; and it 


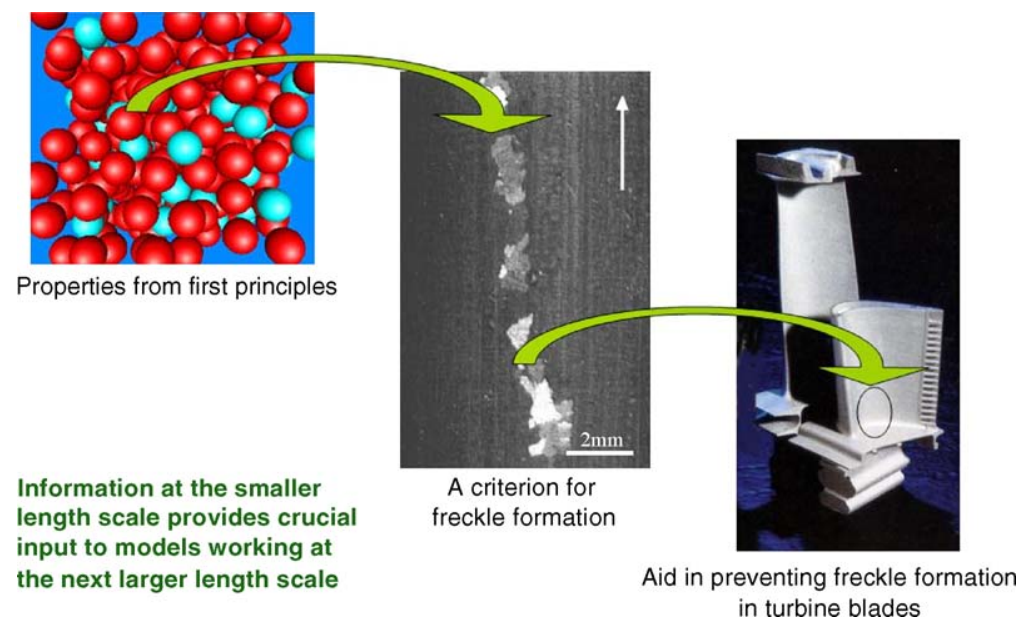

Fig. 1. Schematic diagram of MEANS approach to minimization of freckle formation in turbine blades.

incorporates lifetime prediction and uncertainty modeling. The MEANS program shares much in common with the AIM program (for accelerated insertion of materials) that is supported by the Defense Advanced Research Projects Agency [4].

Ambitious goals have been set for materials, processes, and components that emerge from the MEANS program. Through use of modeling tools and synchronization of materials and systems development, we expect to reduce development times by $\approx 50 \%$. Development costs are expected to drop by $\approx 75 \%$ and operation and service costs to drop by $\approx 33 \%$.

Examples of successful adaptation of MEANS to materials development include: (1) A thermodynamic database has been developed for liquid $\mathrm{Ni}-\mathrm{Al}-\mathrm{X}$ alloys. One goal of this effort is to be able to control mixing instabilities, which can lead to so-called freckle formation [5]. (2) Researchers at Ohio State University and Johns Hopkins University have produced a detailed deformation mechanism map for Ni-based superalloys of interest to aircraft engines [6,7]. Coupled with stress states during service, these maps allow for accurate predictions of performance and lifetime. (3) The MEANS approach is not limited to U.S. research and development. For example, in Japan, Al and $\mathrm{Mg}$ alloys are now being designed by first-principles calculations [8].

\section{Functional materials}

Creation and development of new materials is often, for example, an enabling technology for lasers and optics systems. Here we note three examples of laser programs in the AFOSR portfolio of Dr. Howard Schlossberg and discuss negative-index materials, which fall within the programs of Dr. Gernot Pomrenke and Dr. Harold Weinstock.

\subsection{Solid-state lasers}

The USAF and others have need for compact, high-power lasers. Our efforts address the key issue of gain-to-loss ratios, where energy losses limit the maximum energy at which lasers can be operated (without self-destructing). Ceramic lasers can possess materials properties better than those of single crystals. They have better mechanical properties and, if made properly, better optical properties owing to reduced segregation of alloying elements $[9,10]$. Segregation can be further reduced if the ceramics have nanoscale grain sizes. Other advantages of ceramics over single-crystal hosts include fabrication time, mass reduction, cost, size, and flexibility of design. We envision significant growth in this field of study over the next several years.

\subsection{Mid-IR semiconductor lasers}

Mid-IR semiconductor lasers [11,12] have been the focus of a coordinated research activity within AFRL, in a program brought together at the Directed Energy Directorate in Albuquerque, New Mexico. Their compact sizes (Fig. 2) and wavelengths of operation offer much promise for insertion into AF systems (Fig. 3). The MID-IR wavelength versatility requires very precise control of the quantum-well width.

\subsection{Frequency conversion in lasing}

Frequency conversion has enabled access to otherwise unavailable wavelengths by taking a well-developed laser and converting it to the wavelength needed [13]. A common means by which to accomplish this conversion is to use appropriate crystals to generate harmonics, and accomplish sum or difference frequencies by optical parametric oscillation. Successful materials for conversion include niobates such as $\mathrm{LiNbO}_{3}$, tantalates such as $\mathrm{LiTaO}_{3}$, and GaAs. Breakthroughs have been achieved in the preparation of quasi-phase-matched samples of these materials. The Li-related materials have achieved quasiphase-matching frequency conversion to $5 \mathrm{~W}$ of $532 \mathrm{~nm}$ radiation, for $1000 \mathrm{~h}$ of operation. The GaAs optical parametric oscillator has been tuned by Prof. James S. Harris and his group at Stanford University [14] from 2.3 to $9.1 \mu \mathrm{m}$, with over $50 \%$ photon conversion slope efficiency, by use of lithographically defined periodically oriented thin film templates, overgrown by vapor phase epitaxy. 


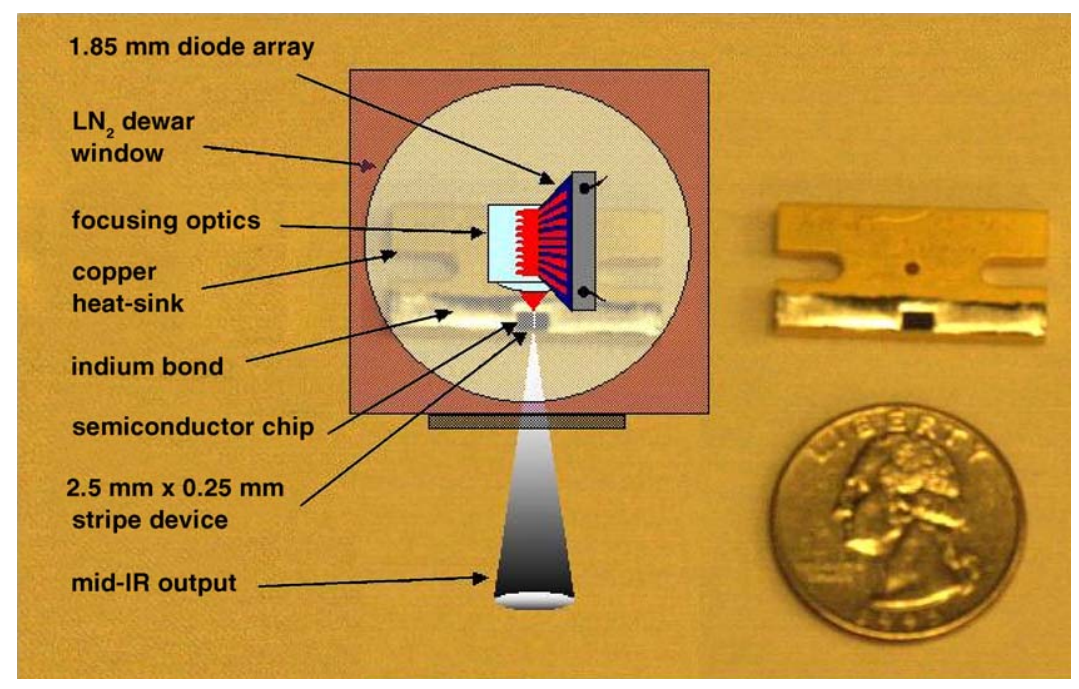

Fig. 2. Design of mid-IR semiconductor laser; note that cooling by liquid nitrogen $\left(\mathrm{LN}_{2}\right)$ is required.

\subsection{Negative-index materials}

It has long been a mathematical oddity that, in principle, mathematical symmetry or reciprocity leave open to conjecture that negative index of refraction materials could exist. However, only recently has realization of this concept been achieved [15-18]. Owing to its unique dispersion, a photonic crystal can give group velocity opposite to phase velocity when electromagnetic waves propagate within it. Such new materials with effective negative refractive index are rewriting the laws of optics and could lead, for example, to a perfect lens, with resolution not limited by wavelength, and to flat-plate optical elements that introduce the concept of "flat cylindrical" lenses. Evanescent waves are amplified through the flat cylindrical lens, so that both propagating and evanescent waves contribute to the resolution of the image, resulting in sub-wavelength resolution. A photonic-crystal flat lens with body-centered-cubic lattice is fabricated layer-by-layer, for two-dimensional and three-dimensional negative-index lenses. The realization of a three-dimensional photonic-crystal lens is not only important for practical applications, but also significant for fundamental research because full three-dimensional negative refraction, as a basic physical phenomenon, has never been experimentally achieved.

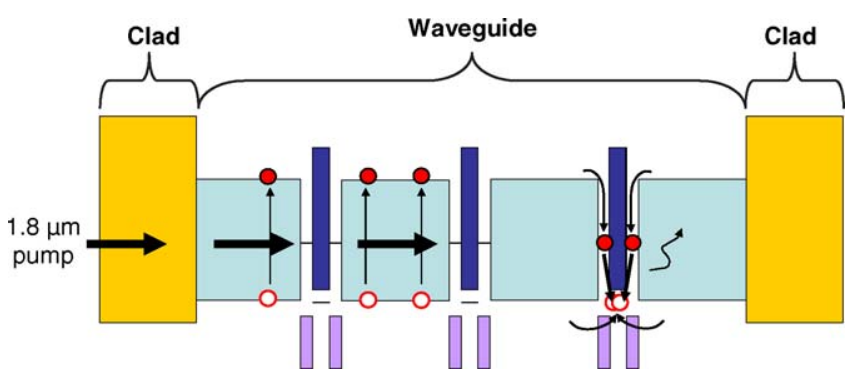

Fig. 3. Operational principle of mid-IR semiconductor laser: pump emission is absorbed in waveguide, creating electron-hole pairs; carriers transfer to quantum wells and recombine to produce photons.
Applications for negative-index materials include compact low-mass antennas. For satellite-to-earth information, intrinsic negative phase shifters that can match impedance to free space and can effect beam steering would be required. Needs to be met include lower losses, unit-cell voltage tuning, and extension to terahertz and IR wavelengths.

\section{Structural materials}

\subsection{High-temperature materials}

The USAF has renewed its interest in hypersonic flight. Hypersonics mandates development and implementation of robust, ultra-high-temperature materials (UHTMs). Ultra-hightemperature ceramics (UHTCs) have advantages of comparatively low density and resistance to environmental degradation $[19,20]$. Conventional processing methods or novel net-shapeforming methods $[21,22]$ may be applied to production of such ceramics.

We require UHTMs that only need to survive for at most a few seconds maximum and for UHTMs that must be affordable, fabricable, reliable, and repairable to serve a wide spectrum of applications (Fig. 4). A simple consideration of speed versus temperature leads to realization that high-Mach propulsion further drives the spectrum of needs, ranging from Mach numbers/ram temperatures of $3.5 / 460{ }^{\circ} \mathrm{C}$, through $5.0 / 1000^{\circ} \mathrm{C}$, to $8 / 2340^{\circ} \mathrm{C}$ and even higher. Above Mach 5 , inlet temperatures surpass use temperatures of currently available materials (metals with ceramic thermal-barrier coatings). Metal/carbide composites, which can withstand high temperatures for limited times with acceptable ablation, exhibit much promise for these applications.

UHTCs for sharp leading edges also hold significant promise. Traditional hypersonic vehicles have been designed with blunt leading edges because of materials limitations. Heating of sharp leading edges is so intense that most materials fail by melting, spalling, or ablating. If suitable materials were to be available, hypersonic vehicles with sharp leading edges would result in 


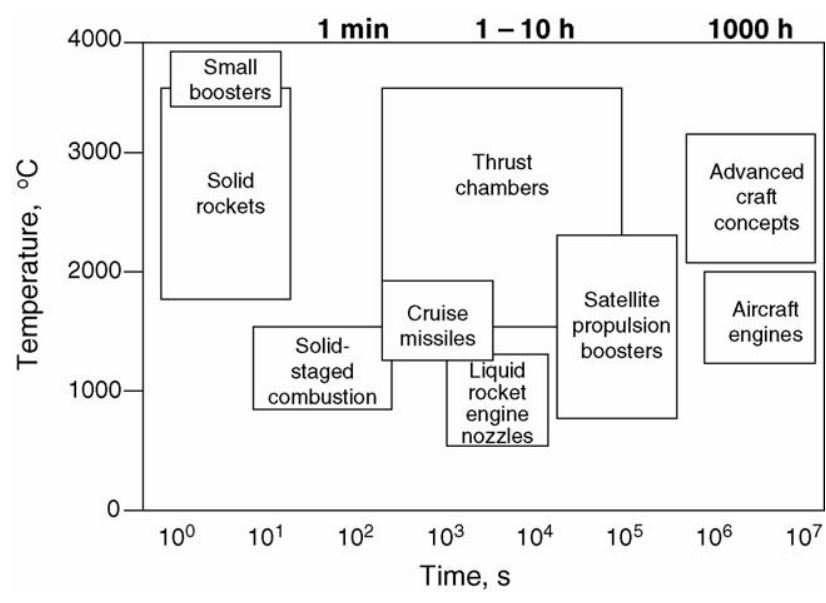

Fig. 4. Range of temperatures and time at temperature for various AF systems.

better lift/drag ratios, cross-range capability, maneuverability at low and high speeds, and landing characteristics. $\mathrm{ZrB}_{2}$ and $\mathrm{HfB}_{2}$ composites are promising candidates for sharp leading edges $[19,20]$. Work in this area is within the program of Dr. Joan Fuller.

\subsection{Self-healing materials}

Although fiber-reinforced polymer-matrix composites exhibit excellent properties and are widely used in aircraft structures, they are difficult and expensive to repair. Microcapsules embedded in the surrounding matrix material can lead to self-healing properties. When a crack in the matrix opens, encapsulated material can react with the surrounding material to rebond the matrix. A crack that would otherwise have continued to grow would be arrested [23-25]. Fig. 5a depicts the three basic goals of fracture- and fatigue-resistant self-healing polymers: increase in static fracture toughness, decrease fatigue sensitivity (slope of curve), and increase in threshold value of applied cyclic load. Experiments on epoxy-based polymers have confirmed attainment of these goals. Fig. 5b shows results of cohesive modeling of the crack retardation observed in a self-healing polymer.
Future work will concentrate on the potential for enhanced performance with different scale sizes, distribution geometry, and other physical properties. Work is also being supported in new, highly tough nanocomposites $[26,27]$. These projects fall within the program of Dr. Byung-Lip (Les) Lee.

\section{Mathematical guidance}

Simulation and modeling have come to occupy a large fraction of the materials research supported by AFOSR. The MEANS paradigm dictates validation of these models through appropriate experimentation. Select examples of modeling efforts follow.

\subsection{Theory of imperfectly bonded suspensions}

Theoretical treatment of imperfectly bonded suspensions of particles has led to surprising findings that bear on setting realistic expectations [27-29]. Here we consider a problem posed by the Materials Laboratory within AFRL, where inclusions dispersed throughout a host material might debond or experience some sort of chemical surface reaction, such as corrosion, and the newly created thin region thus produced has its own thermal conductivity which, as theoretical work by Lipton has shown, could result in an aggregate conductivity that differs from what one would have anticipated [30]. In some cases, the aggregate conductivity was the same as that of the host material, the addition of the inclusions with the thin debonded or corroded region having no impact. This work falls under the program of Dr. Arje Nachman.

\subsection{Computer design of materials for bio/nanosensing}

Work by Dr. Ruth Pachter at the AFRL Materials Laboratory has achieved striking success at design of materials through use of theoretical models. Predicted properties have been confirmed by parallel experiments [31]. Using density functional theory, and applying recent advances in the prediction of optical absorption, she and her colleagues have successfully predicted spectra for reverse-saturable absorption materials, based on calcula-

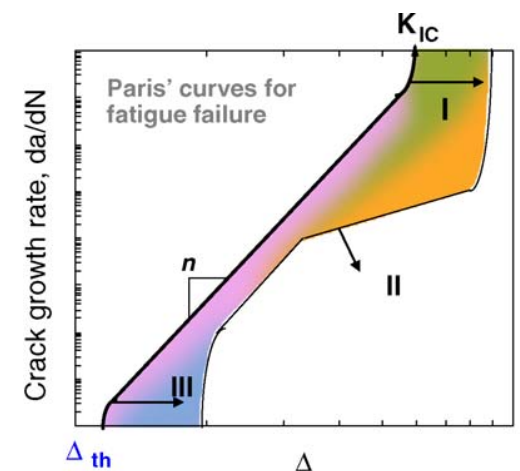

(a)

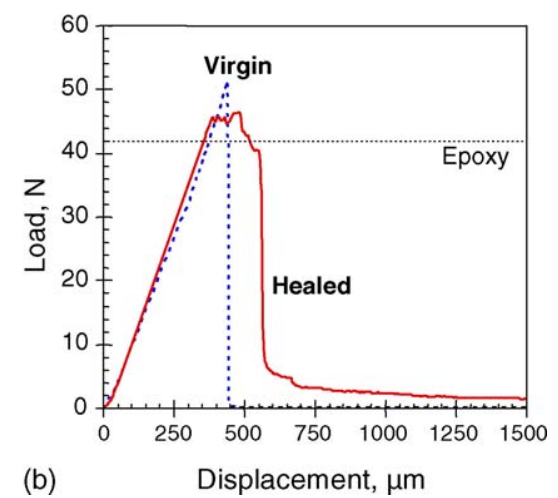

(b)

Fig. 5. For self-healing polymers: (a) schematic representation of crack growth rate vs. change in stress-intensity factor $(\Delta K)$, where $\Delta K_{\text {th }}$ is the threshold value and $\Delta K_{\mathrm{IC}}$ is the critical factor for Mode I loading; (b) load-displacement curves for conventional and self-healing epoxy-based specimens. 
tions of excited state cross sections. Optical spectra predictions, with application to sensor material design, have shown excellent agreement over a range of energies. These results were obtained directly from ab initio calculations, demonstrating the growing power of ab initio work for complex molecules built of multiatom constituents.

She and her group have also performed computer simulations for biological chromophores, applying a multi-scale approach to enable design of biochromophores with required optical absorption for green fluorescent protein. In common with the ab initio calculations for complex molecules built from multiatoms is that the computer modeling led to excellent agreement between the calculated and observed experimental results. What is entirely different is that for the biological system ab initio calculations were impossible. In contrast, instead, a statistical or imperical model, not physical model, had to be constructed as the foundation for the model calculations. A multi-scale approach for molecular dynamics simulations, with periodic boundary conditions, led to the theoretical calculations based on empirical relationships for the biological system properties.

These physical-molecular and empirical-biological modeling successes point the way to greater new horizons for the promise and role of computer modeling in design of future materials.

\subsection{Tailoring materials with selected properties}

An AFRL-sponsored research team discovered in 2005 aluminum (Al) atom clusters that, when reacted with iodine, exhibit chemical properties similar to those of the single atoms of metal- lic and nonmetallic elements. The finding is significant because it reveals the potential of an enhanced chemistry based on a new periodic table of cluster elements. Scientists can use this expanded chemistry to create unique compounds with distinctive new properties. Dr. Shiv Khanna of Virginia Commonwealth University [32] and his university colleagues are responsible for this breakthrough, supported by two AFOSR program managers.

This work began with altering atom clusters of one element, $\mathrm{Al}$, without changing its number of protons and electrons. The goal was to induce a cluster to behave the same way that a single atom of another element behaves. The work theoretically and experimentally examined chemical properties, electronic structures, and geometries of Al clusters in chemical compounds containing iodine atoms. Energy calculations to determine the bonding mechanism between the Al cluster and an iodine atom revealed bonding based on an electron localized within the $\mathrm{Al}$ cluster, showing the cluster to maintain its own integrity throughout the reaction. They then substituted Al clusters for iodine atoms in naturally occurring chains of iodine atoms in molecules known as polyiodides. The demonstrated flexibility of clusters of Al to act as individual iodine atoms has added a new dimension to exploring the traditional periodic table of elements, with cluster compounds of $\mathrm{Al}(13)$ clusters behaving like iodine atoms, $\mathrm{Al}(14)$ clusters behaving like alkaline-earth metals, and raising the question of creating a series of clusters to build a new "periodic table," not of elements but of clusters simulating properties of elements: clusters as building blocks to tailor the design of future nanoscale materials with designer properties. Work in

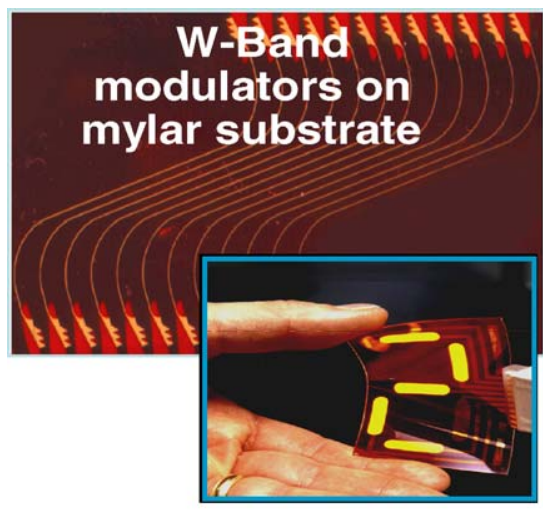

Flexible lightemitting diode

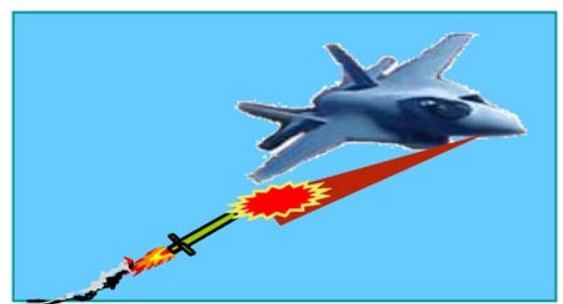

Conformal smart skin
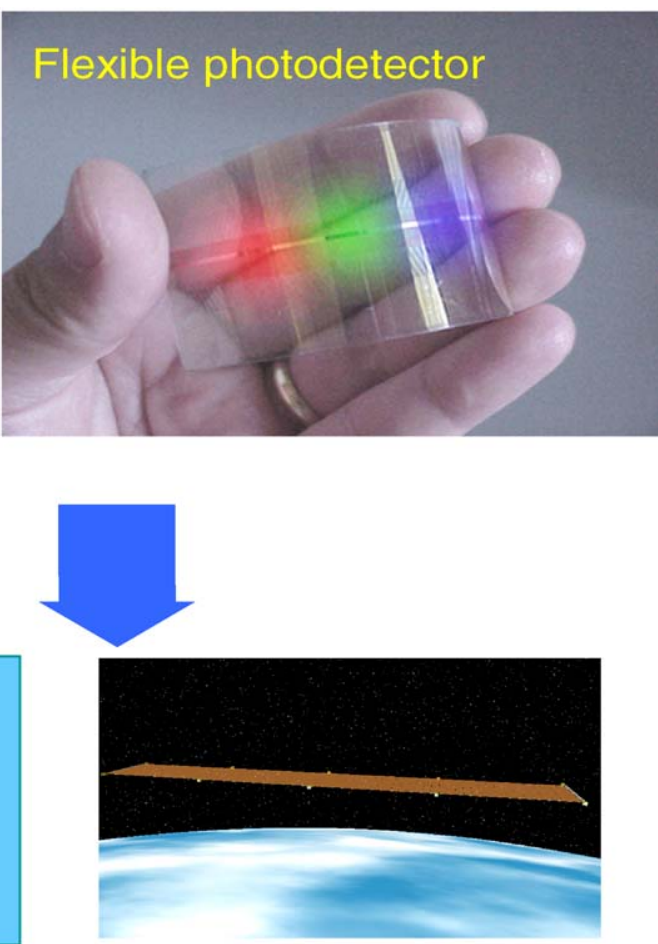

Plastic sensor carpet

Fig. 6. Variety of applications for flexible, multifunctional polymers; this work is guided by Dr. Charles Lee of AFOSR. 
this area was in the programs of Dr. Gernot Pomrenke and Dr. Michael Berman.

\section{Concluding remarks}

The examples above provide a snapshot of materials research now being funded by AFOSR. They have, for clarity of presentation, been divided into classes. AFOSR strongly believes, however, in design and use of multifunctional materials (Fig. 6). We are compelled to support the needs of further, faster, longer, with greatly increased capability. Multifunctional materials and components will often be required to accomplish our missions. The illustrations presented here will, we hope, direct your interest to the exciting work is progress. It has been described at a level of depth and detail appropriate to a broad review. Readers are encouraged to seek further information, through the appended references or by contact with the researchers who have been cited.

\section{Acknowledgments}

We thank the referenced AFOSR Program Managers and our contractors for their valued assistance in putting this material together.

\section{References}

[1] http://www.afosr.af.mil// and links therein.

[2] http://www.acq.osd.mil/sadbu/sbir/.

[3] http://www.afosr.af.mil/pdfs/Hartley/MEANSInfo.pdf (as of 15 October 2005).

[4] http://www.darpa.mil/dso/thrust/matdev.aim.htm (as of 15 October 2005).

[5] C. Frueh, D.R. Poirier, R.G. Erdmann, S.D. Felicelli, Mater. Sci. Eng. A 345 (2003) 72-80.

[6] G.B. Viswanathan, P.M. Sarosi, M.F. Henry, D.D. Whitis, W.W. Milligan, M.J. Mills, Acta Mater. 53 (2005) 3041-3057.
[7] G.B. Viswanathan, P.M. Sarosi, D.D. Whitis, M.J. Mills, Mater. Sci. Eng. A 400-4001 (2005) 489-495.

[8] H. Somekawa, T. Tanaka, H. Sasaki, K. Kita, A. Inoue, K. Higashi, Acta Mater. 52 (2004) 1051-1059.

[9] A. Ikesue, Opt. Mater. 19 (2002) 183-187.

[10] V. Lupei, A. Lupei, A. Ikesue, J. Alloys Compd. 380 (2004) 61-70.

[11] H.K. Choi, Curr. Op. Sol. State Mater. Sci. 1 (1996) 212-217.

[12] K.D. Moiseev, M.P. Mikhailova, Y.P. Yakovlev, Phys. E 20 (2004) 491-495.

[13] G.P.A. Malcolm, M. Ebrahimzadeh, A.I. Ferguson, IEEE J. Quant. Electon. 28 (1992) 1172-1178.

[14] http://snowmass.stanford.edu/ (as of 15 October 2005).

[15] R.A. Shelby, D.R. Smith, S. Schultz, Science 292 (2001) 77-79.

[16] D.R. Smith, W.J. Padilla, D.C. Vier, C. Nemat-Nasser, S. Schultz, Phys. Rev. Lett. 84 (2000) 4184-4187.

[17] T. Decoopman, T. Crepin, M. Perrin, S. Fasquel, A. Marteau, X. Melique, E. Lheurette, O. Vanbesien, D. Lippins, C. R. Physique 6 (2005) 683-692.

[18] P.W. Milonni, G.J. Maclay, Opt. Commun. 228 (2003) 161-165.

[19] S.R. Levine, E.J. Opila, M.C. Halbig, J.D. Kiser, M. Singh, J.A. Salem, J. Eur. Ceram. Soc. 22 (2002) 2757-2767.

[20] M.M. Opeka, I.G. Talmy, E.J. Wuchina, J.A. Zaykowski, S.J. Causey, J. Eur. Ceram. Soc. 19 (1999) 2405-2414.

[21] P. Kumar, N.A. Travitzky, P. Beyer, K.H. Sanhage, R. Janssen, N. Claussen, Scripta Mater. 44 (2001) 751-757.

[22] K.H. Sandhage, P. Kumar, U.S. Patent 6,407,022 (2002).

[23] M.R. Kessler, N.R. Sottos, S.R. White, Comp. Part A 34 (2003) 743-753.

[24] M.R. Kessler, S.R. White, Comp. Part A 32 (2001) 683-699.

[25] M.J. Tari, A. Bals, J. Park, M.Y. Lin, H.T. Hahn, Comp. Part A 29 (1998) 651-661.

[26] C.H. Yang, H. Huh, H.T. Hahn, Int. J. Sol. Struct. 42 (2005) 6141-6165.

[27] R. Lipton, B. Vernescu, Math. Meth. Mod. Appl. Sci. 5 (1995) $1139-1173$

[28] R. Lipton, B. Vernescu, J. Appl. Phys. 79 (1996) 8964-8966.

[29] R. Lipton, B. Vernescu, Proc. R. Soc. Lond. A 452 (1996) 329-358.

[30] R. Lipton, Louisiana State University, Private communication, June 2005.

[31] R. Pachter, Wright-Patterson Air Force Base, Private communication, June 2005.

[32] D.E. Bergeron, A.W. Castleman Jr., N.O. Jones, S.N. Khanna, Chem. Phys. Lett. 415 (2005) 230-233 